\title{
Allopurinol metabolism in a patient with xanthine oxidase deficiency
}

\author{
HISASHI YAMANAKA, KUSUKI NISHIOKA, TAKEHIKO SUZUKI, * \\ AND KEIICHI KOHNO* \\ From the Rheumatology Department, Clinical Research Centre, Tokyo Women's Medical College, 2-4-1, NS \\ BLD, Nishishinjuku, Shinjukuku, Tokyo, Japan
}

SUMMARY A patient with complete deficiency of xanthine oxidise would not be expected to oxidase allopurinol to oxipurinol if allopurinol did not have any alternative metabolic pathway. 400 mg of allopurinol was administered to a patient with xanthine oxidase deficiency, and plasma allopurinol, oxipurinol, hypoxanthine, and xanthine levels were determined serially by the use of high-performance liquid chromatography (HPLC). Plasma oxipurinol as well as allopurinol was increased after the administration of allopurinol, and oxipurinol reached a maximum level of $13 \cdot 1$ $\mu \mathrm{g} / \mathrm{ml}$ at 6 hours after the administration. This was the same pattern as that of normal controls. This result demonstrated the existence of some other oxidising enzyme of allopurinol than xanthine oxidase.

Allopurinol (4-hydroxypyrazolo[3;4-d]pyrimidine) is known as a potent inhibitor of xanthine oxidase (EC 1.2.3.2). In addition allopurinol itself is metabolised in vivo by xanthine oxidase to oxipurinol (4,6-dihydroxypyrazolo[3,4-d]pyrimidine, which also inhibits xanthine oxidase. A patient with complete deficiency of xanthine oxidase would not be expected to oxidise allopurinol to oxipurinol in vivo, if allopurinol did not have any alternative metabolic pathway. In this paper, we report the conversion of allopurinol to oxipurinol in a patient with deficiency of xanthine oxidase and discuss the possible metabolic pathway.

\section{Materials and methods}

The patient ${ }^{1}$ was a 54 -year-old female. She had been suffering from Graves's disease since she was 36 years old and had received methimazole continuously, but there was no history of haematuria, abdominal colicky pain, urinary calculi, or administration of allopurinol. Her parents were consanguineous and her sister had chronic thyroiditis. At the age of 49 hypouricaemia was revealed by chance. Her serum uric acid level was only $0.4 \mathrm{mg} / \mathrm{dl}(0.02 \mathrm{mmol} / \mathrm{l})$. On the other hand her urinary excretion of oxypurine, determined by the method of Jørgensen and Poulsen, ${ }^{2}$ was as high as $320 \mathrm{mg} /$ day on a purine-

Accepted for publication 7 January 1983.

*Products Formulation Research Laboratory, Tanabe Seiyaku, Osaka, Japan.

Correspondence to Dr K. Nishioka. free diet. Creatinine clearance was $73.3 \mathrm{ml} / \mathrm{min}$, and no calculus was found in her urinary tract by abdominal plain $x$-ray film and drip infusion pyelography.

The xanthine oxidase activity of the duodenal mucosa, which was obtained by gastrofibroscopy, was determined by the following procedures. The tissue specimens, weighing 5 to $10 \mathrm{mg}$, were homogenised in sodium pyrophosphate buffer solu-

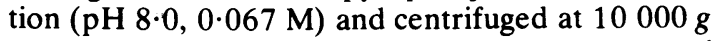
for 40 minutes. The supernatants were concentrated and then reacted with a reaction mixture $(\mathrm{pH} 8.0)$ containing $0.5 \mathrm{mM}$ of hypoxanthine at $37^{\circ} \mathrm{C}$ for 30 minutes, and the uric acid product was determined by the use of a spectrophotometer at $292 \mathrm{~nm}$. Protein concentrations were determined by the method of Lowry et al. ${ }^{3}$

No activity of xanthine oxidase was found in this patient compared with that of a normal control, which was $15.4 \mathrm{nmol} / \mathrm{hour} / \mathrm{mg}$ protein. She was diagnosed as having complete deficiency of xanthine oxidase.

$400 \mathrm{mg}$ of allopurinol was administered orally in one dose to this patient. At the same time $150 \mathrm{mg}$ of allopurinol was administered to 2 healthy men whose serum uric acid levels were within normal limits as the controls. Plasma samples of this patient and controls were obtained serially by heparinised venous puncture until 25 hours after the administration of allopurinol.

Plasma allopurinol, oxipurinol, hypoxanthine and xanthine were determined by the following methods. Allopurinol was commercially obtained from Tanabe Seiyaku (Oaska, Japan). Oxipurinol was supplied 
from the same company. Xanthine was obtained from Sigma (St Louis, Missouri, USA), and hypoxanthine from Kohjin (Tokyo, Japan). Chelex-100 resin (200-400 mesh) was obtained from Bio-Rad Laboratories (Richmond, California, USA). All other chemicals were of analytical reagent grade commercially available.

Plasma concentrations of allopurinol, oxipurinol, hypoxanthine, and xanthine were determined by the method of Suzuki et al. (paper in preparation) by a modified procedure of Brown and Bye. ${ }^{4}$ Chelex $-100 \mathrm{Cu}^{++}$resins were prepared according to the method of Brown et al., ${ }^{4}$ packed into glass columns $(0.6 \times 25 \mathrm{~cm})$. and washed with water followed by $0.02 \mathrm{M}$ carbonate buffer, $\mathrm{pH} 11 \cdot 0$. Aliquots $(0 \cdot 2$ $\mathrm{ml}$ ) of plasma were applied to the columns. The columns were washed with carbonate buffer, $\mathrm{pH} 11 \cdot 0$, followed by water, and eluted with $1 \mathrm{M}$ ammonium solution. The eluate was collected in a volumetric flask, which contained $0.2 \mathrm{ml}$ of internal standard solution (sodium salicylate), and filled up to $10 \mathrm{ml}$. The resultant mixture was lyophilised to dryness, and the residue was then dissolved in $400 \mu$ l of water. A $100 \mu \mathrm{l}$ aliquot of the solution was injected into the high-performance liquid chromatography (HPLC) system. Chromatography was performed with a 0.39 $\times 30 \mathrm{~cm} \mu$ Bondapak $\mathrm{C} 18$ reversed-phase column and $0.01 \mathrm{M}$ ammonium phosphate $(\mathrm{pH} 6.0)$ as the mobile phase. A detector wavelength of $254 \mathrm{~nm}$ was used. This method was suitable for analysing oxypurines in the range of $0.05-20 \mu \mathrm{g}$ in $0.2 \mathrm{ml}$ of plasma. Recoveries of the 4 compounds of interest were $85-95 \%$.

\section{Results}

The plasma allopurinol and oxipurinol of this patient and healthy controls are shown in Fig. 1. In the control group the concentration of plasma allopurinol was highest at 2 hours after the oral administration of allopurinol and then rapidly decreased. The concentration of plasma oxipurinol was highest at 8 hours after administration of the allopurinol and then decreased gradually. In the patient the maximum plasma allopurinol level was observed at $1 \frac{1}{2}$ hours after its administration, after which it rapidly decreased. Oxipurinol increased in the same pattern as that of the controls, reaching the value of $13 \cdot 1$ $\mu \mathrm{g} / \mathrm{ml}$ at 6 hours after administration, of the allopurinol.

Plasma hypoxanthine and xanthine concentrations are shown in Fig. 2. In the controls almost no significant change was seen, but in the patient hypoxanthine was markedly decreased between 6 and 8 hours after the administration of allopurinol, simultaneously with the peak level of plasma oxipurinol.

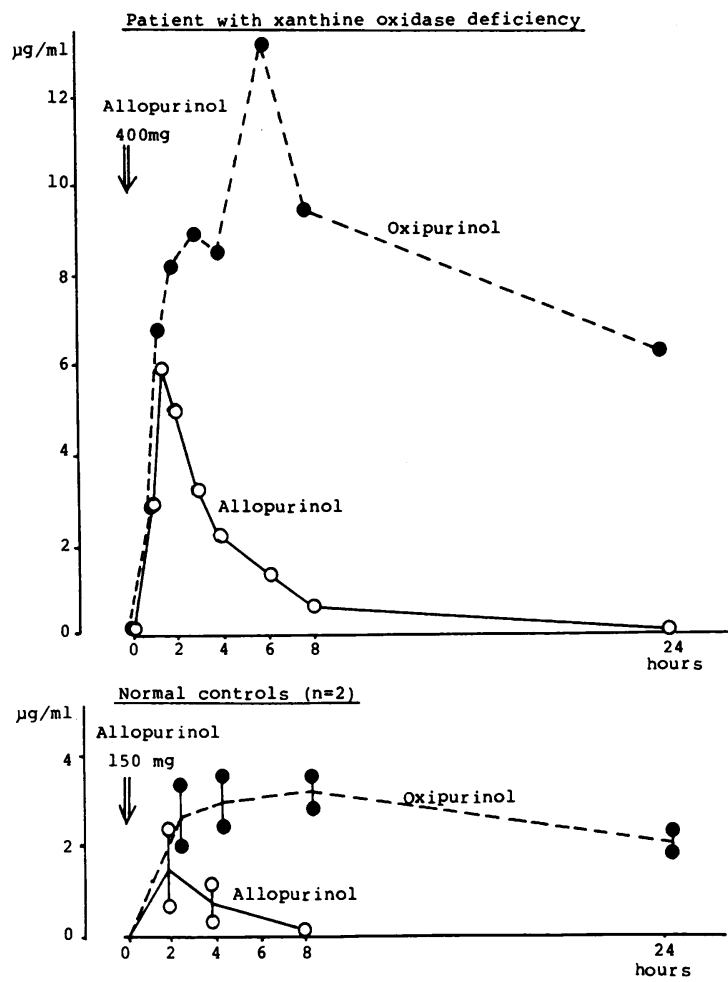

Fig. 1 Allopurinol and oxipurinol after the oral adminsistration of allopurinol.

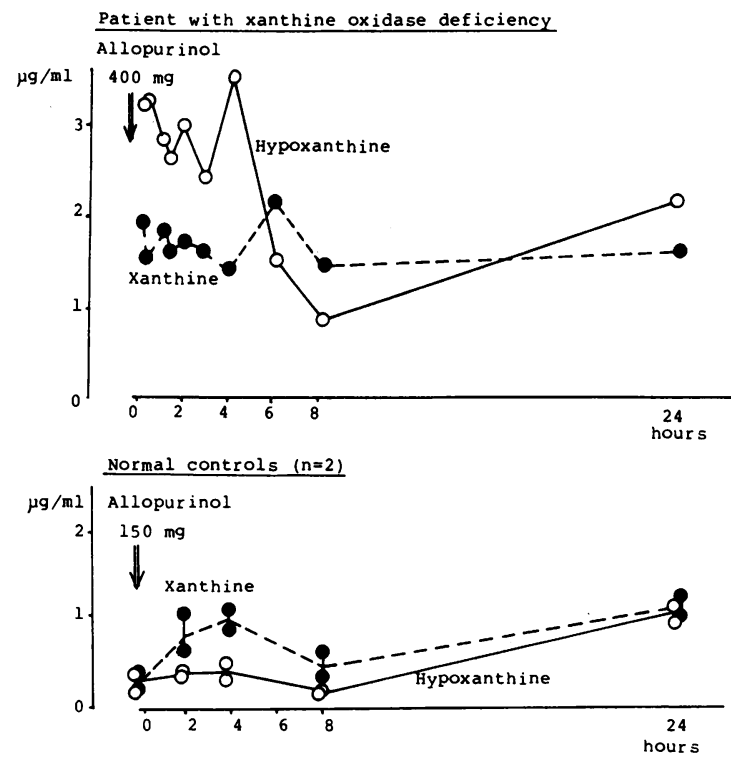

Fig. 2 Hypoxanthine and xanthine after the oral administration of allopurinol. 


\section{Discussion}

In connection with the metabolism of allopurinol in the patient with xanthinuria it was of interest to analyse whether or not some enzyme other than xanthine oxidase might be present. There have been previous reports on the urinary excretion of allopurinol and oxipurinol when allopurinol was administered to patients with xanthinuria, but the results conflicted greatly. Various authors ${ }^{5-8}$ reported that alopurinol was excreted unchanged and that oxipurinol was absent in the urine of their cases. Others ${ }^{2-11}$ observed the conversion of allopurinol to oxipurinol in the urine of their xanthinuric patients. In view of this finding some enzyme that oxidises allopurinol even in the absence of xanthine oxidase might be present. Chalmers et al. ${ }^{10}$ suggested inosinic acid dehydrogenase (EC 1.2.1.14) as this alternative enzyme, but later Nelson et al. ${ }^{12}$ took exception to this because allopurinol ribotide could not be a substrate for this enzyme. Then Johns et al. ${ }^{13}$ and others suggested that aldehyde oxidase (EC 1.2. 3. 1.) had the capacity to oxidise allopurinol to oxipurinol. Yamamoto et al. ${ }^{14}$ noted that acute intoxication by allopurinol was observed to be greater in female than in male mice and concluded that some testosterone-dependent enzyme to oxidise allopurinol was present. Finally to support this conclusion they reported that it was aldehyde oxidase.

In this report we attempted to prove the existence of some enzyme other than xanthine oxidase in allopurinol metabolism. We analysed allopurinol and oxipurinol in the plasma of a patient with complete deficiency of xanthine oxidase by the use of HPLC and investigated the possibility of an alternative enzyme other than xanthine oxidase taking part in allopurinol metabolism. The plasma concentration of oxipurinol of this patient increased in the same pattern as that of the normal controls, and the biological half lives of allopurinol and oxipurinol showed almost the same values in these 2 groups.

This fact indirectly suggested the existence of another enzyme than xanthine oxidase. Furthermore, we must accept the possibility that allopurinol is oxidised by this other enzyme even in normal subjects

Patients with xanthinuria may differ in their enzyme patterns. Further investigation by direct methods will be needed to clarify this.

\section{References}

1 Katsuki T, Shimizu T, Nishina T, Fujihira K, Nishioka K, Ohsawa T. A case of asymptomatic xanthine oxidase deficiency. Nippon Naika Gakkai Zasshi 1981; 70: 1263-6.

2 Jørgensen S, Poulsen H E. Enzymatic determination of hypoxanthine and xanthine in human plasma and urine. Acta Pharmacol 1955; 11: 223-43.

3 Lowry O H, Rosebrough N J, Farr A L, Randall R J. Protein measurement with the folin phenol reagent. J Biol Chem 1951; 193: 265-75.

4 Brown $M$, Bye A. The determination of allopurinol and oxipurinol in human plasma and urine.J Chromotogr 1977; 142: 195-202.

5 Engelman K, Watts R W E, Klinenberg J R, Sjoerdsma A, Seegmiller J E. Clinical physiological and biochemical studies of a patient with xanthinuria and pheochromocytoma. Am J Med 1964 37: 839-61.

6 Elion G B, Kovensky A, Hitchings G H, Metz E, Rundles R W. Metabolic studies of allopurinol, an inhibitor of xanthine oxidase. Biochem Pharmacol 1966; 15: 863-80.

7 Simmonds H A, Levin B, Cameron J S. Variations in allopurinol metabolism by xanthinuric subjects. Clin Sci Mol Med 1974; 47: 173-8.

8 Holmes E W, Mason D H, Goldstein L I, Blount R E, Kelley W N. Xanthine oxidase deficiency: studies of a previously unreported case. Clin Chem 1974; 20: 1076-9.

9 Simmonds H A. Urinary excretion of purines, pyrimidines and pyrazolopyrimidines in patients treated with allopurinol or oxipurinol. Clin Chim Acta 1969; 23: 353-64.

10 Chalmers R A, Parker R, Simmonds H A, Sneddon W, Watts $R$ W E. The conversion of 4-hydroxypyrazolo[3,4-d] pyrimidine (allopurinol) into 4,6-dihydroxypyrazolo[3,4d] pyrimidine (oxipurinol) in vivo in the absence of xanthine-oxygen oxidore ductase. Biochem J 1969; 112: 527-32.

11 Auscher C, Pasquier C, Mercier N, Delbarre F. Oxidation of pyrazolo[3,4-d]pyrimidine in a xanthinuric man. Isr J Med Sci 1973; 9: 1082.

12 Nelson D J, Bugge C J L, Krasny H C, Elion G B. Formation of nucleotides of $\left[6^{-14} \mathrm{C}\right]$ allopurinol and $\left[6-{ }^{14} \mathrm{C}\right]$ oxipurinol in rat tissues and effects in uridine nucleotide pools. Biochem Pharmacol 1973; 22: 2003-22.

13 Johns D G. Human liver aldehyde oxidase: Differential inhibition of oxidation of charged and uncharged substrates. J Clin Invest 1967; 46: 1492-505.

14 Yamamoto I, Kyo K, Iwata H. A study of the enzyme metabolizing allopurinol (author's translation) Uric Acid Research 1973; 1: 21-33. 